\title{
SIMULTANEOUS LOCALIZATION AND MAP BUILDING ALGORITHM FOR REAL-TIME APPLICATIONS
}

\author{
Stefano Panzieri* Federica Pascucci* \\ Roberto Setola**
}

\author{
* Dip. Informatica e Automazione, Università "Roma \\ Tre", Via della Vasca Navale, 79, 00144, Roma, Italy \\ ** Università CAMPUS Biomedico - Facoltà di Ingegneria, \\ Via E. Longoni, 86, 00153 Rome, Italy
}

\begin{abstract}
Mobile robot navigation in unstructured environment is a challenging task due to the uncertain nature of the real world. Navigating using visual landmarks could be a mandatory skill together with the ability of building a representation of the world around the robot. This mapping aptitude should be implemented as an efficient real-time task, even if a large number of elements have to be included in the map itself. To this aim, and to help in localising the robot, a promising technique is given by the Extended Kalman Filter in its interlaced version. The resulting SLAM algorithm, proposed in this paper, has a reduced computational cost preserving, at the same time, a good performance. Copyright (C) 2005 IFAC
\end{abstract}

Keywords: Kalman filters, Mobile robots, Visual Motion, Real Time, Extended Kalman filters, SLAM

\section{INTRODUCTION}

When mobile robots operate in real world environments they require both reliable localisation systems, and a sufficiently precise map of the working area. Thus, most successful mobile robot systems include a localisation module together with an a priori knowledge of the environment (i.e., a map) in their control architecture. The localisation module, by means of the map, is able to provide a reliable estimation of the robot position.

A more challenging problem arises when "both the map and the robot location are unknown. In this case, the robot starts in an unknown position, in an unknown environment, and tries to incrementally build a map while using the same map to compute its pose in the environment" (Clark S. et al., 2001). This problem is referred in literature as simultaneous localisation and map building (SLAM), and several approaches have been investigated to solve it after the seminal paper of (Smith R.C. and Cheeseman P., 1986). The solution of SLAM has been addressed with approaches based on Bayesian filtering (Guivant J. and Nebot E., 2001; Clark S. et al., 2001). These techniques approximate the probability representation using samples of probability density distributions (Thrun S. et al., 1998). Although they are still computationally expensive for real time applications, they present significant advantages in solving the data association problem.

In this framework, one of the most appealing approaches to solving real time SLAM problems uses the well known predictor-corrector structure of the Kalman filter: a solution is given by modeling the environment and the sensors, and assuming 
that errors have a Gaussian distribution (Gelb A.C., 1994). The measures of the proprioceptive sensory system of the vehicle (e.g., encoders, gyros) are used to compute a raw location estimation, while, the exteroceptive sensors refine this estimation and update the map.

Drawbacks of such approach are memory requirements and computational loads that quadratically increase with the number of map objects (beacons). In densely populated environments, the number of beacons detected will make those needs to be beyond the power capabilities of computer resources.

In this paper, we propose a Kalman based solution to SLAM, suitable for real-time implementations. Indeed, our algorithm has a memory occupancy and a computational load linearly proportional to the number of beacons detected by the sensory system. In particular, a vision system is interfaced with a mobile robot allowing the use of ceiling lamps as natural landmarks. A brief description of the image processing algorithm will be given in section 2. In section 3 we review the interlaced Kalman filter introduced in (Glielmo L. et al., 1999), while in section 4 we describe its application to the SLAM case. Its reduced computational cost is evaluated in section 5 and some experiments, whose results are shown in section 6 , conclude the paper.

\section{VISION SYSTEM}

As stated above, the vision system presented in this work should be able to recognise artificial sources of light during the navigation of a mobile robot in an office like environment.

The camera is mounted focusing the ceiling such that the distance between the image plane and the landmarks along the focal axis is fixed and known. This reduce the overall complexity of features extraction, because also the size of the landmarks is fixed.

The image processing algorithm developed follows the fundamental steps of image analysis: preprocessing, segmentation, feature extraction.

\section{- Preprocessing}

Assuming that the beacon have a frame representation with large luminance values, a threshold is applied to obtain a binary image. The key function of the threshold operation is to improve the image in way that increases chances for the success of other processes: indeed, in this step small reflection are partially removed cause their areas normally decrease.

- Segmentation

After thresholding, the image is partitioned into its constituents objects, performing a graph search of all connected components (blobs). The segmentation step usually retrieves more than one blob, and, sometimes reflections can produce small connected components that can be discriminated evaluating their area.

- Feature extraction

After segmentation the position of the lamps in the image plane is obtained applying the well known formulas for calculating the centre of mass. In order to improve the quality of this shape, a binary morphological operator is applied on the connected components. An opening operation with a circular structuring element is performed to smooth the contour end eliminate protrusions.

\section{IEKF FILTER}

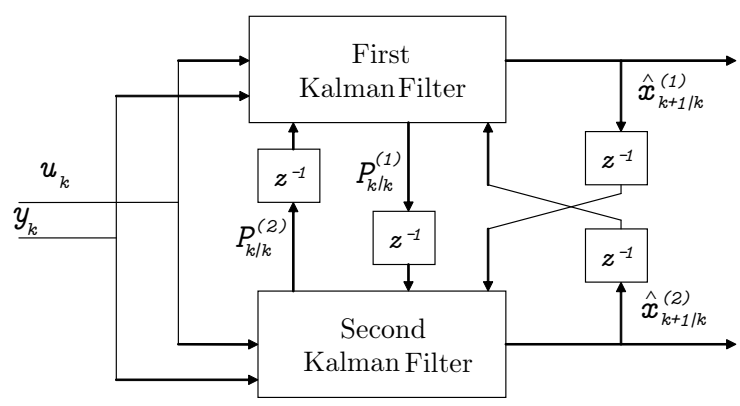

Fig. 1. Interlaced Extended Kalman Filter

The Interlaced Extended Kalman Filter (IEKF) has been proposed in (Glielmo L. et al., 1999) to reduce computational load of the estimation process for a class of nonlinear system. The fundamental idea of the IEKF derived from the multiplayers dynamic game theory, where the solution of the game is such that each player chooses its strategy as optimal response to the strategy chosen by the other players. In the context of estimation the players are the estimation algorithm, the strategy is the estimate, the object function is a measure of the covariance estimation error. In particular IEKF consists of $m$ parallel implementation of Kalman Filters (KF). Each KF works independently by the others and is designed to estimate a subset of the state variables, considering the remaining parts as deterministic time varying parameters. The error introduced is partially alleviated increasing the noise covariance matrices.

For sake of simplicity, let us consider a system whose state vector can be partitioned into just two subsets, can be put in the form

$$
\begin{aligned}
{\left[\begin{array}{c}
x_{k+1}^{(1)} \\
x_{k+1}^{(2)}
\end{array}\right] } & =\left[\begin{array}{cc}
A^{(1)} & \mathbf{0} \\
\mathbf{0} & A^{(2)}
\end{array}\right]\left[\begin{array}{l}
x_{k}^{(1)} \\
x_{k}^{(2)}
\end{array}\right]+ \\
& +\left[\begin{array}{l}
f^{(1)}\left(x_{k}^{(2)}, u_{k}\right) \\
f^{(2)}\left(x_{k}^{(1)}, u_{k}\right)
\end{array}\right]+\left[\begin{array}{l}
\xi_{k}^{(1)} \\
\xi_{k}^{(2)}
\end{array}\right]
\end{aligned}
$$


where the state vector $x \in \mathcal{R}^{n}$ has been partitioned into $x^{(1)} \in \mathcal{R}^{n_{1}}$ and $x^{(2)} \in \mathcal{R}^{n_{2}}$ (with $\left.n=n_{1}+n_{2}\right), f^{(i)}$ are differentiable functions, and $\xi_{k}^{(i)} \in \mathcal{R}^{n_{i}}, i=1,2$ are zero-mean uncorrelated white process noise vectors characterized by the covariance matrices $Q_{k}^{(i)}, i=1,2$ and $u_{k}$ is the input vector.

Further, the system is assumed to have an output equation that can be put into the following equivalent form

$$
\begin{aligned}
& y_{k}=C^{(1)}\left(x_{k}^{(2)}\right) x_{k}^{(1)}+D^{(1)}\left(x_{k}^{(2)}\right)+\psi_{k} \\
& y_{k}=C^{(2)}\left(x_{k}^{(1)}\right) x_{k}^{(2)}+D^{(2)}\left(x_{k}^{(1)}\right)+\psi_{k}
\end{aligned}
$$

where $\psi_{k} \in \mathcal{R}^{m}$, are zero-mean uncorrelated white measurement noise vector characterized by the covariance matrices $R_{k}$.

Under this hypothesis, the first filter of the IEKF computes the estimate $\hat{x}_{k \mid k}^{(1)}$ using the predictive estimate $\hat{x}_{k \mid k-1}^{(2)}$ obtained, at the previous step, by the other filter. Notice that, after replacing $x_{k}^{(2)}$ with $\hat{x}_{k \mid k-1}^{(2)}$ in (1) and (2) the first subsystem can be considered as a linear time varying system dependent on the known input $f^{(1)}\left(\hat{x}_{k \mid k-1}^{(2)}, u_{k}\right)$. Similar considerations holds for the second subsystem.

Each $\mathrm{KF}$ is characterised by the following equations (for the first filter $i=1$ and $j=2$, while for the second $i=2$ and $j=1$ ):

$$
\begin{aligned}
\tilde{Q}_{k}^{(i)}= & Q_{k}^{(i)}+J_{x, j}^{f, i} P_{k-1 \mid k-1}^{(j)} J_{x, j}^{f, i}+J_{u}^{f, i} Q_{k}^{u} J_{u}^{f, i^{T}}(3 \mathrm{a}) \\
P_{k \mid k-1}^{(i)}= & A^{(i)} P_{k-1 \mid k-1}^{(i)} A^{(i) T^{T}}+\tilde{Q}_{k}^{(i)} \\
\tilde{R}_{k}^{(i)}= & R_{k}+C^{(j)}\left(\hat{x}_{k \mid k-1}^{(i)}\right) P_{k \mid k-1}^{(j)} C^{(j)}\left(\hat{x}_{k \mid k-1}^{(i)}\right)^{T}(3 \mathrm{c}) \\
K_{k}^{(i)}= & P_{k \mid k-1}^{(i)} C^{(i)}\left(\hat{x}_{k \mid k-1}^{(j)}\right)^{T}\left[C^{(i)}\left(\hat{x}_{k \mid k-1}^{(j)}\right) P_{k \mid k-1}^{(i)} \times\right. \\
& \left.\times C^{(i)}\left(\hat{x}_{k \mid k-1}^{(j)}\right)^{T}+\tilde{R}_{k}^{(i)}\right]^{-1} \\
\hat{x}_{k \mid k}^{(i)}= & \hat{x}_{k \mid k-1}^{(i)}+K_{k}^{(i)}\left[y_{k}-C^{(i)}\left(\hat{x}_{k \mid k-1}^{(j)}\right) \hat{x}_{k \mid k-1}^{(i)}+\right. \\
& \left.+D^{(i)}\left(\hat{x}_{k \mid k-1}^{(j)}\right)\right] \\
\hat{x}_{k+1 \mid k}^{(i)}= & A^{(i)} \hat{x}_{k \mid k}^{(i)}+f^{(i)}\left(\hat{x}_{k \mid k-1}^{(j)}, u_{k}\right) \\
P_{k \mid k}^{(i)}= & P_{k \mid k-1}^{(i)}-K_{k}^{(i)} C^{(i)}\left(\hat{x}_{k \mid k-1}^{(j)}\right) P_{k \mid k-1}^{(i)}
\end{aligned}
$$

where $J_{x, j}^{f, i}$ is the Jacobian of $f^{(i)}$ w.r.t. $x^{(j)}$, $P_{k \mid k-1}^{(i)}$ is the covariance matrix of the estimation error variable $e_{k \mid k-1}^{(i)}:=x_{k}^{(i)}-\hat{x}_{k \mid k-1}^{(i)}$ for $i=1,2$. From (3a) and (3c) one can notice that the process and measurement noise covariance matrices $Q_{k}^{(i)}$ and $R_{k}$ are suitable increased by addition of positive semi definite quantities that take into account the error introduced by the decoupling operation. Indeed $\tilde{Q}_{k}^{(i)}$ and $\tilde{R}_{k}^{(i)}$ represent, respectively, the process and measurement covariance of the "modified" error

$$
\begin{aligned}
\tilde{\xi}_{k}^{(i)} & :=\xi_{k}^{(i)}+J_{x, j}^{f, i} e_{k \mid k-1}^{(i)}+J_{x, j}^{f, i} u_{k} \\
\tilde{\psi}_{k}^{(i)} & :=\psi_{k}+C^{(j)}\left(\hat{x}_{k \mid k-1}^{(i)}\right) e_{k / k-1}^{(i)} .
\end{aligned}
$$

The proposed formulation of IEKF assumes that state transition mapping and the observation mapping depend linearly and affinely on their argument. If one removes these assumptions, the algorithm can be still applied by linearising each part of the system in the neighbourhood of the estimations obtained by the other filters at the previous step.

\section{IEKF SLAM FILTER}

In this paper we use IEKF filter to solve the SLAM problem. In this framework we assume that all uncertainty sources have unimodal Gaussian distribution and provide a model for the robot, the beacon positions, and the sensors. The mobile platform considered is a robot with the kinematics of an unicycle. The robot is equipped with encoders and gyro, as proprioceptive sensors, while uses the vision system presented above as exteroceptive sensor. The measurement provided by the exteroceptive sensory system are expressed in the robot coordinates and represent the position of the beacons in the viewing windows of the web cam. Under the SLAM framework, the filter is able to localise the robot and concurrently build a simple geometric map (a list of beacon positions). During the navigation task, the system detects new features when exploring new areas. Once those feature become reliable, they are included into the map.

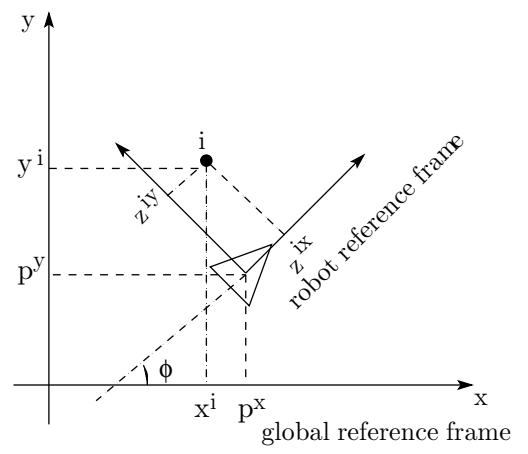

Fig. 2. Reference frames used in SLAM filter

\subsection{Complete system model}

The state of the whole system at the $k$-th sampling interval is composed by the configuration of the 
robot together with the positions of all beacons w.r.t. a global reference frame (see Fig. 2):

$$
x_{k}=\left(x_{k}^{r T}, x_{k}^{b^{T}}\right)^{T} .
$$

Define the robot state vector as

$$
x_{k}^{r}=\left(p_{k}^{x}, p_{k}^{y}, \phi_{k}, b_{k}\right)^{T}
$$

where $b$ is the gyro bias, and define the inputs for the robot model as

$$
u_{k}=\left(\delta s_{k}, \omega_{k}\right)^{T}
$$

where $\delta s_{k}$ is the vehicle displacement and $\omega_{k}$ its angular velocity during the $k$-th sampling interval.

The robot dynamic is modelled using the equation of the unicycle model:

$$
\begin{aligned}
x_{k}^{r} & =f\left(x_{k-1}^{r}, u_{k}\right)= \\
& =\left[\begin{array}{llll}
1 & 0 & 0 & 0 \\
0 & 1 & 0 & 0 \\
0 & 0 & 1 & -\delta t_{k} \\
0 & 0 & 0 & 1
\end{array}\right] x_{k-1}^{r}+\left[\begin{array}{ccc}
\cos \tilde{\phi}_{k} & 0 \\
\sin \tilde{\phi}_{k} & 0 \\
0 & 1 \\
0 & 0
\end{array}\right] u_{k}
\end{aligned}
$$

where $\tilde{\phi}_{k}=\phi_{k-1}+\left(\omega_{k}-b_{k-1} \delta t_{k}\right) / 2$ is the average robot orientation during the sampling time interval $\delta t_{k}$.

The beacon state vector is defined as

$$
x_{k}^{b}=\left[p_{k}^{1}, \ldots, p_{k}^{N}\right]^{T}
$$

where $p_{k}^{i}=\left(x_{k}^{i}, y_{k}^{i}\right)$ is the location of the $i$-th beacon in the global reference frame. The state transition equation for the beacons can be written as

$$
x_{k}^{b}=x_{k-1}^{b}
$$

since beacons are assumed to be static. Notice that the size of $x_{k}^{b}$ is dynamically increased any time a new beacon appears in the camera image.

The observation equation describes the relation between robot configuration and position of beacons in the viewing windows of the web cam (referred as active beacon in from now on). The observation vector

$$
z_{k}=h\left(x_{k}\right)
$$

consists of sub vectors $z_{k}^{i}, i=1, \ldots, M$, where $M$ is the number of active beacons and

$$
z_{k}^{i}=h^{i}\left(x_{k}\right)=\left[\begin{array}{c}
z_{k}^{i x} \\
z_{k}^{i y}
\end{array}\right]=\mathbf{R}_{k}^{\phi}\left[\begin{array}{c}
x_{k}^{i} \\
y_{k}^{i}
\end{array}\right]-\mathbf{R}_{k}^{\phi}\left[\begin{array}{c}
p_{k}^{x} \\
p_{k}^{y}
\end{array}\right]
$$

being $\mathbf{R}_{k}^{\phi}$ the rotation matrix between the robot reference frame and the global frame reference (see Fig. 2)

$$
\mathbf{R}_{k}^{\phi}=\left[\begin{array}{cc}
\cos \phi_{k} & \sin \phi_{k} \\
-\sin \phi_{k} & \cos \phi_{k}
\end{array}\right]
$$

Due to the structure of the system model, IEKF can be adopted partitioning the system into $2+M$ subsystem:

- robot position subsystem estimating $p_{k}^{r}=$ $\left(p_{k}^{x}, p_{k}^{y}\right)$

- robot orientation subsystem estimating $\theta_{k}=$ $\left(\phi_{k}, b_{k}\right)$

- $M$ beacon position subsystems each one estimating a beacon position $p_{k}^{i}=\left(x_{k}^{i}, y_{k}^{i}\right)$

\subsection{Robot position subsystem}

The robot dynamics can be written in the form

$$
p_{k}^{r}=p_{k-1}^{r}+f^{r}\left(z_{k-1}, u_{k}, \delta t_{k}\right)+\xi_{k-1}^{r}
$$

where $\xi_{k}^{r} \in \mathcal{R}^{2}$ is a zero-mean white noise vector with covariance matrix $Q_{k}^{r}$ and

$$
f^{r}\left(\theta_{k-1}, u_{k}, \delta t_{k}\right)=\left[\begin{array}{cc}
\cos \tilde{\phi}_{k} & 0 \\
\sin \tilde{\phi}_{k} & 0
\end{array}\right] u_{k}
$$

The observation vector is composed by $M$ sub vectors

$$
z_{k}^{r, i}=C^{r}\left(\theta_{k}\right) p_{k}^{r}+D^{r}\left(p_{k}^{(i)}, \theta_{k}\right)+\psi_{k}^{(i)}
$$

where $\psi_{k}^{(i)} \in \mathcal{R}^{2}$ is a zero-mean white noise vector with covariance matrix $R_{k}^{(i)}$ and

$$
C^{r}\left(\theta_{k}\right)=-\mathbf{R}_{k}^{\phi} ; \quad D^{r}\left(p_{k}^{(i)}, \theta_{k}\right)=\mathbf{R}_{k}^{\phi} p_{k}^{(i)} .
$$

\subsection{Robot orientation subsystem}

The orientation dynamic $\phi$ and the gyro bias $b$ may be formulated as

$$
\theta_{k}=A^{\theta} \theta_{k-1}+f^{\theta}\left(u_{k}\right)+\xi_{k-1}^{\theta}
$$

where $\xi_{k}^{\theta} \in \mathcal{R}^{2}$ is a zero-mean white noise vector with covariance matrix $Q_{k}^{\theta}$ and

$$
A^{\theta}=\left[\begin{array}{cc}
1 & -\delta t_{k} \\
0 & 1
\end{array}\right] ; \quad f^{\theta}=\left[\begin{array}{ll}
0 & 1 \\
0 & 0
\end{array}\right] u_{k}
$$

The output equation consists of $M$ sub vector

$$
z_{k}^{\theta, i}=h^{\theta}\left(\theta, p^{r}, p^{(i)}\right)+\psi_{k}^{\theta}
$$

where $h^{\theta}\left(\theta, p^{r}, p^{(i)}\right)$ is a nonlinear mapping

$$
h^{\theta}\left(\theta, p^{r}, p^{(i)}\right)=\mathbf{R}_{k}^{\phi}\left(p_{k}^{(i)}-p_{k}^{r}\right)
$$




\subsection{Beacon position subsystem}

The state transition model of each beacon is

$$
p_{k}^{(i)}=p_{k-1}^{(i)}+\xi_{k-1}^{(i)} ; \quad i=1, \ldots, M
$$

where $\xi_{k}^{(i)} \in \mathcal{R}^{2}$ is a zero-mean white noise vector with covariance matrix $Q_{k}^{(i)}$

The associated output equations result in

$$
z_{k}^{(i)}=C^{(i)}\left(\theta_{k}\right) p_{k}^{(i)}+D^{(i)}\left(p_{k}^{r}, \theta_{k}\right)+\psi_{k}^{(i)}
$$

where

$$
C^{(i)}\left(\theta_{k}\right)=\mathbf{R}_{k}^{\phi} ; \quad D^{(i)}\left(p_{k}^{r}, \theta_{k}\right)=-\mathbf{R}_{k}^{\phi} p_{k}^{r}
$$

In order to reduce the number of parallel subsystems, we decide to pack all the $M$ beacon filters in the active beacon subsystem. At $k$-th sampling time the active beacon are retrieved and each sub vectors is used to dynamically compose the state vector of such filter. For example if the beacons $i$ and $j$ are in the viewing windows of the web cam, the active beacon subsystem become:

$$
\begin{aligned}
& {\left[\begin{array}{c}
p_{k}^{(i)} \\
p_{k}^{(j)}
\end{array}\right]=\left[\begin{array}{c}
p_{k-1}^{(i)} \\
p_{k-1}^{(j)}
\end{array}\right]+\left[\begin{array}{c}
\xi_{k-1}^{(i)} \\
\xi_{k-1}^{(j)}
\end{array}\right]} \\
& {\left[\begin{array}{c}
z_{k}^{(i)} \\
z_{k}^{(j)}
\end{array}\right]=\left[\begin{array}{cc}
\mathbf{R}_{k}^{\phi} & \mathbf{0} \\
\mathbf{0} & \mathbf{R}_{k}^{\phi}
\end{array}\right]\left[\begin{array}{c}
p_{k}^{(i)} \\
p_{k}^{(j)}
\end{array}\right]-\left[\begin{array}{cc}
\mathbf{R}_{k}^{\phi} & \mathbf{0} \\
\mathbf{0} & \mathbf{R}_{k}^{\phi}
\end{array}\right]\left[\begin{array}{c}
p_{k}^{r} \\
p_{k}^{r}
\end{array}\right]}
\end{aligned}
$$

\section{COMPUTATIONAL COST}

It is well known that classical SLAM algorithms have computational cost and memory requirement proportional to $\sim O\left(N^{2}\right)$, being $N$ the number of the states used to represent all the beacons and the vehicle position.

In order to reduce the computational load in (Guivant J. and Nebot E., 2001) the authors propose CSLAM. This algorithm segments the map into a set of fixed disjointed areas and solves the SLAM problem only with respect to the beacons present inside one area (i.e. where there is the vehicle). Full update of the filter is performed only when the vehicle move away from the area. Further, to reduce memory occupation, in (Guivant J. and Nebot E., 2002), the authors introduce a de- coupling procedure in CSLAM nullifying the correlation terms related with beacons belonging to different constellations. In this way, memory and computational requirements of the algorithm are proportional to $\sim O\left(N \cdot N_{b}\right)$, where $N_{b}$ is the number of beacons present inside to each area.

As noted before, our algorithm stores separately information related to each filter. Then it is im- mediate to recognise that the memory occupancy is proportional to

$$
M_{R} \sim 4+4+4 \cdot N
$$

that can be approximated as $M_{R} \sim O(N)$.

On the other side, the computational load of the filters for position and orientation of the vehicle is proportional to $\sim O\left(4+4+M^{2}\right)$, where $M$ is the number of beacons present inside the camera image. However, notice that almost all the equations of the position filter, excepted the ones devoted to calculate the augmented process and measurement covariance matrices $\tilde{R}_{k}^{r}$ and $\tilde{Q}_{k}^{r}$, have a cost proportional to a dimension four (the same also for the orientation filter neglecting the calculations requested to compute $\tilde{R}_{k}^{\theta}$ and $\left.\tilde{Q}_{k}^{\theta}\right)$.

During the estimation phase, the filters associated with the beacons present inside the camera image are assembled into a single object. The computational load of this filter is proportional to $\sim O\left(4+4+M^{2}\right)$, but if we neglect the calculation requested to compute $\tilde{R}_{k}^{b}$, it is proportional to $\sim O\left(M^{2}\right)$.

In any case, because $M \ll N$ (but also $M<N_{b}$ ), we archive a considerable reduction of computation load.

\section{EXPERIMENTAL RESULTS}

Experimental trials have been carried out using a robotised wheelchair prototype built at the robotics lab of the University of "Roma Tre" and a Philips Vesta Pro Scan. The vehicle has two driving wheels equipped with low resolution incremental encoders (6.4 pulses $/ \mathrm{mm}$ of the wheel movement).

The proprioceptive sensory system is completed by a piezoelectric gyro (MuRata), that measures rotation velocity. The gyro has a good accuracy $(3 \%)$ but is affected by temperature depending bias.

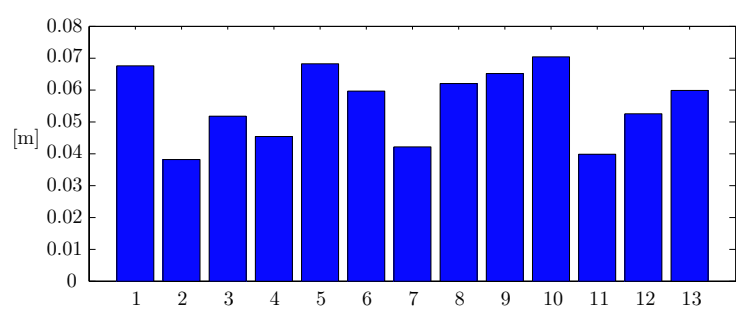

Fig. 3. Landmark estimation errors

The software implementation is based on two notebooks connected over an Ethernet link. The first laptop installed on the wheelchair runs the control software. A data acquisition card (DAQPad 1200 by National Instruments) interfaces the 
sensory and driving systems under a LabVIEW application that includes some $\mathrm{C}$ routines for the time critical tasks of the filter implementation. The second laptop, is devoted to process images from the vision system including a web cam mounted on the robot and focusing the ceiling. The distance from the lights is about $2.50 \mathrm{~m}$ and each pixel is thereafter about $5 \mathrm{~mm}$ large at the CIF resolution $(352 \times 288)$.

The results our experiment are reported in Figs. 3 and 4 . The robot describes a double 8-path in an office-like environment and the camera is able to view only few beacons (zero to three) for each frame. The estimated beacons positions are shown in Fig. 4 as red stars and the distance from their exact position is reported in Fig. 3. The statistical values reported in table 6 show that the quality of the estimate is almost the same for each beacon due to the decoupled nature of the filter.

The localisation results are shown in Fig. 4 where the odometric path, as estimated on the basis of encoders and gyro data, is compared with the filter output.

Table 1. Map error (in $\mathrm{cm}$ )

\begin{tabular}{|c|c|c|c|}
\hline $\begin{array}{c}\text { Mean } \\
\text { value }\end{array}$ & $\begin{array}{c}\text { Standard } \\
\text { deviation }\end{array}$ & $\begin{array}{c}\text { Min } \\
\text { error }\end{array}$ & $\begin{array}{c}\text { Max } \\
\text { error }\end{array}$ \\
\hline \hline 5.5 & 1.14 & 3.8 & 7 \\
\hline
\end{tabular}

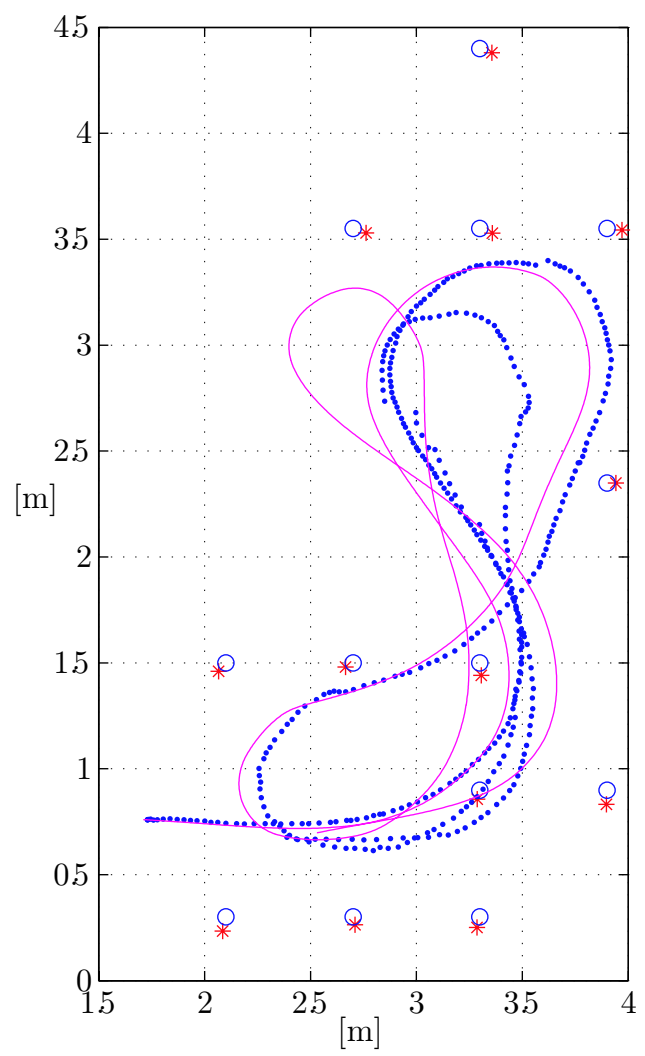

Fig. 4. Corrected path (blue), odometry (magenta), and landmark estimated position $(*)$ vs. real (o)
Finally, we remark that, using the same experimental test bed, the execution time of the proposed filter is $60 \%$ less than the algorithm reported in (Panzieri S. et al., 2003).

\section{CONCLUSIONS}

This paper describes an algorithm based on a modified version of EKF for SLAM problems. Specifically, the peculiar structure of the problem at hand allows the use of the Interlaced version (IEKF). This algorithm represents a good tradeoff between accuracy and computational load. In particular, it has been shown that computational load and memory capacity requested by IEKF increase at least linearly with the number of beacons (while clasical approches have a quadratic dependency).

Further work will be devoted to test IEKF in other configurations (e.g., outdoor), with different testbeds and in the presence of a multitude of mobile robots.

\section{REFERENCES}

Clark S., Dissanayake G., Newman P. and Durrant-whyte H.F. (2001). A solution to simultaneous localization and map building (slam) problem. IEEE Trans. on Robotics and Automation vol. 17(no 3), 229-241.

Gelb A.C. (1994). Applied Optimal Estimation. MIT Press. Cambridge,MA.

Glielmo L., Setola R. and Vasca F. (1999). An interlaced extended kalman filter. IEEE Trans. on Automatic Control vol. 44(no 8), 15461549 .

Guivant J. and Nebot E. (2001). Optimization of simultaneous localization and map building algorithm for real time implementation. IEEE Trans. on Robotics and Automation vol.17(no 3), 242-256.

Guivant J. and Nebot E. (2002). Improving computational and memory requirements of simultaneous localization and map building algorithm. In: Proc. of 2002 IEEE Int. Conf. on Robotics and Automation. Washington DC.

Panzieri S., Pascucci F. and Ulivi G. (2003). Vision based navigation using kalman approach for slam. In: Proc. of 11th Int. Conf. on Advanced Robotics. Coimbra, Portugal.

Smith R.C. and Cheeseman P. (1986). On the representation and estimation of spatial uncertainty. Int. J. of Robotics Research vol. 5(no 4), 56-68.

Thrun S., Fox D. and Burgard W. (1998). A probabilistic approach to concurrent mapping and localization for mobile robots. March learning Autonom Robots vol.31, 29-53. 